\title{
Cognitive Performance, Physical Pain and Psychological Distress in Women with Fibromyalgia During The COVID-19 Pandemic in Chile: A Case- Control Study
}

\section{Miguel Ramos-Henderson \\ Universidad Santo Tomás \\ Ignacio Toro-Roa \\ Universidad Santo Tomás \\ Rocío Aguilera-Choppelo \\ Universidad Santo Tomás}

\section{Diego Palominos}

Catholic University of the North

Marcio Soto-Añari

Universidad Católica San Pablo

Norman Lopez

University of the Coast

Marcos Domic-Siede ( $\square$ mdomic@ucn.cl)

Catholic University of the North

\section{Research Article}

Keywords: Fibromyalgia (FMS), cognitive impairment, executive functions, COVID-19.

Posted Date: September 7th, 2021

DOI: https://doi.org/10.21203/rs.3.rs-866271/v1

License: (a) (i) This work is licensed under a Creative Commons Attribution 4.0 International License. Read Full License 


\section{Abstract}

Fibromyalgia (FMS) is a chronic condition that encompasses widespread pain associated with cognitive impairment and significant emotional distress related to functional disability that affects mainly women. However, the mechanisms underlying the origin of the cognitive impairments and how the current COVID19 pandemic impacts remain elusive. This study aimed to determine whether there is an association between cognitive performance in executive functions, physical pain, and psychological distress caused by the pandemic in a group of Chilean women with FMS using a novel online protocol of neuropsychological evaluation. We found that women with FMS present cognitive impairment predominantly explained by pain and anxiety symptoms, which are associated with the difficulties experienced from the COVID-19 pandemic. These results point out the importance of considering a broader perspective for treatment, including anxiety symptoms and the psychological distress caused by the pandemic as a therapeutic target for cognitive impairments.

\section{Introduction}

Fibromyalgia (FMS) is a chronic syndrome characterized by complex symptomatology, the core of which is generalized and persistent musculoskeletal pain that also comprises fatigue, sleep disturbances, morning joint stiffness, depression, and anxiety ${ }^{1,2}$. This syndrome is one of the most common causes of widespread chronic pain and is a common condition in the general population ${ }^{3,4}$. The worldwide prevalence of FMS is between $2 \%$ and $5 \%$, regardless of the territory, and it mainly affects women ${ }^{5}$. Despite lacking studies in Chile, a similar prevalence is estimated, with predominance in women as well 6 . On the other hand, FMS has been associated with the suffering of physical and emotional trauma and a history of post-traumatic stress ${ }^{7,8}$. Its impact on quality-of-life compromises physical, psychological, and social domains ${ }^{9,10}$, including performing adequately in the work context ${ }^{11}$.

Additionally, cognitive impairments in FMS patients have been reported, such as forgetfulness, concentration difficulties and/or mental slowness. These impairments are known as fibrofog and are considered among the most severe symptoms of the disease ${ }^{12,13}$. Several affected cognitive functions have been identified in a variety of studies, such as attention and memory ${ }^{14,15}$, and cognitive processing speed ${ }^{15}$. Furthermore, executive function impairment has also been confirmed, including planning ${ }^{16}$, abstract thinking ${ }^{17}$, and cognitive flexibility ${ }^{18}$. In this line, a recent meta-analysis that included 37 FMS case studies and controls concluded a decrease in the performance of memory, attention, processing speed, and mainly executive functions ${ }^{19}$.

The mechanisms underlying the origin of the aforementioned impairments are not well understood yet. However, the Neurocognitive Model of Attention to Pain might provide a theoretical framework to understand and test whether the pain interferes with attention processes. According to this model, any cognitive dysfunction in the context of chronic pain is the result of the interference between attention focused on both the peripheral input (pain) and the goal-directed activity ${ }^{20,21}$. In other words, pain and 
high interoceptive monitoring might lead to reduced attention performance in cognitively demanding tasks or activities, in turn increasing the susceptibility to distraction and slowing down the information processing, interfering with cognitive function ${ }^{21}$.

Additionally, there is a susceptibility to suffering from anxiety and depressive disorders in patients with FMS ${ }^{22,23}$, which can increase the reported cognitive deficits ${ }^{24}$. In this line, there is a crucial link between cognitive decline, pain, and psychological distress.

Currently, the emergency due to COVID-19 has seriously impacted the population's mental health ${ }^{25}$. In this context, it has been reported that FMS patients who experience chronic pain present a higher risk of increased psychological distress ${ }^{26}$. However, how the pandemic and its potential effect on the mental health of FMS patients is related to their cognitive performance remains elusive. Besides, there is a lack of these types of studies on the Latin American population, which might be of particular interest considering both the socio-cultural aspects and that the response to the pandemic has been slower and with fewer resources than developed countries ${ }^{27}$.

Here we aimed to determine whether there is a relationship between cognitive performance, physical pain, and psychological distress in Chilean women with FMS during the COVID-19 pandemic using a novel online protocol of neuropsychological evaluation. In order to do this, a group of FMS patients and controls were contacted and evaluated using online platforms in an asynchronous way first, where they answered a sociodemographic scale, pain level rating, psychological distress, and executive functions assessment using behavioral scales. Afterward, they were contacted to conduct a synchronic online evaluation with trained neuropsychologists using traditional virtually adapted cognitive tests (Fig. 1).

We hypothesize that FMS patients present higher levels of psychological distress due to the pandemic and conjointly with physical pain; in agreement with the Neurocognitive Model of Attention to Pain, it will negatively impact their cognitive functions. Our results will contribute to the scientific body of knowledge and the clinical field since they may impact the development of clinical guidelines and health personnel training. Likewise, it is possible to promote and develop public policies that benefit patients with FMS, considering that there is still an under-consideration of these cases ${ }^{28}$.

\section{Results}

We found no significant differences between groups in sociodemographic characteristics. However, there were higher scores in depressive symptoms, panic attacks, and pharmacological treatment in the clinical dimension of our sample. Table 1 shows a summary of the sociodemographic and clinical descriptive statistics. 
Table 1

Clinical and Sociodemographic Characteristics of FMS and Control Groups ${ }^{a}$

\begin{tabular}{|c|c|c|c|c|c|c|}
\hline \multirow{3}{*}{ Characteristic } & \multirow{2}{*}{$\begin{array}{l}\text { FMS } \\
\mathrm{N}=\mathbf{5 1}\end{array}$} & & \multicolumn{2}{|c|}{ Control } & \multirow[b]{3}{*}{$p$} & \multirow[b]{3}{*}{$d / V$} \\
\hline & & & $N=19$ & & & \\
\hline & $M / N$ & SD / \% & $M / N$ & SD / \% & & \\
\hline Age & 44.05 & 8.71 & 40.63 & 9.79 & 0.16 & -.38 \\
\hline Education (in years) & 13.74 & 4.68 & 13.89 & 5.21 & 0.90 & -03 \\
\hline \multicolumn{7}{|l|}{ Time since diagnosis } \\
\hline Less than a year & 2 & 2.85 & - & - & & \\
\hline 1 year & 4 & 5.71 & - & - & & \\
\hline 2 years & 13 & 18.57 & - & - & & \\
\hline 3 years & 6 & 8.57 & - & - & & \\
\hline More than 3 years & 26 & 37.14 & - & - & & \\
\hline \multicolumn{7}{|l|}{ Marital status } \\
\hline Single & 13 & 25.49 & 10 & 52.63 & 0.08 & .34 \\
\hline Married & 15 & 29.41 & 7 & 36.84 & & \\
\hline Divorced / Separated & 14 & 27.45 & 1 & 5.26 & & \\
\hline Widowed & 1 & 1.96 & 0 & 0.00 & & \\
\hline Cohabitating & 8 & 15.68 & 1 & 5.26 & & \\
\hline \multicolumn{7}{|l|}{ Occupation } \\
\hline Unemployed & 9 & 17.64 & 2 & 10.52 & 0.26 & .27 \\
\hline Retired & 6 & 11.76 & 0 & 0.00 & & \\
\hline Housework & 12 & 23.52 & 3 & 15.78 & & \\
\hline Active w/ medical license & 7 & 13.72 & 3 & 15.78 & & \\
\hline Active w/o medical license & 17 & 33.33 & 11 & 57.89 & & \\
\hline \multicolumn{7}{|l|}{ House and family } \\
\hline Single & 2 & 3.92 & 1 & 5.26 & 0.84 & .14 \\
\hline
\end{tabular}

${ }^{a} p<0.05^{\star}, p<0.001^{\star \star} . M=$ Mean. SD = Standard Deviation. $d=$ Cohen's $d . V=$ Cramer's V. FMS = Fibromyalgia Syndrome, CVA = cerebrovascular accident, $\mathrm{OCD}=$ Obsessive Compulsive Disorder, PTSD = Posttraumatic Stress Disorder. 


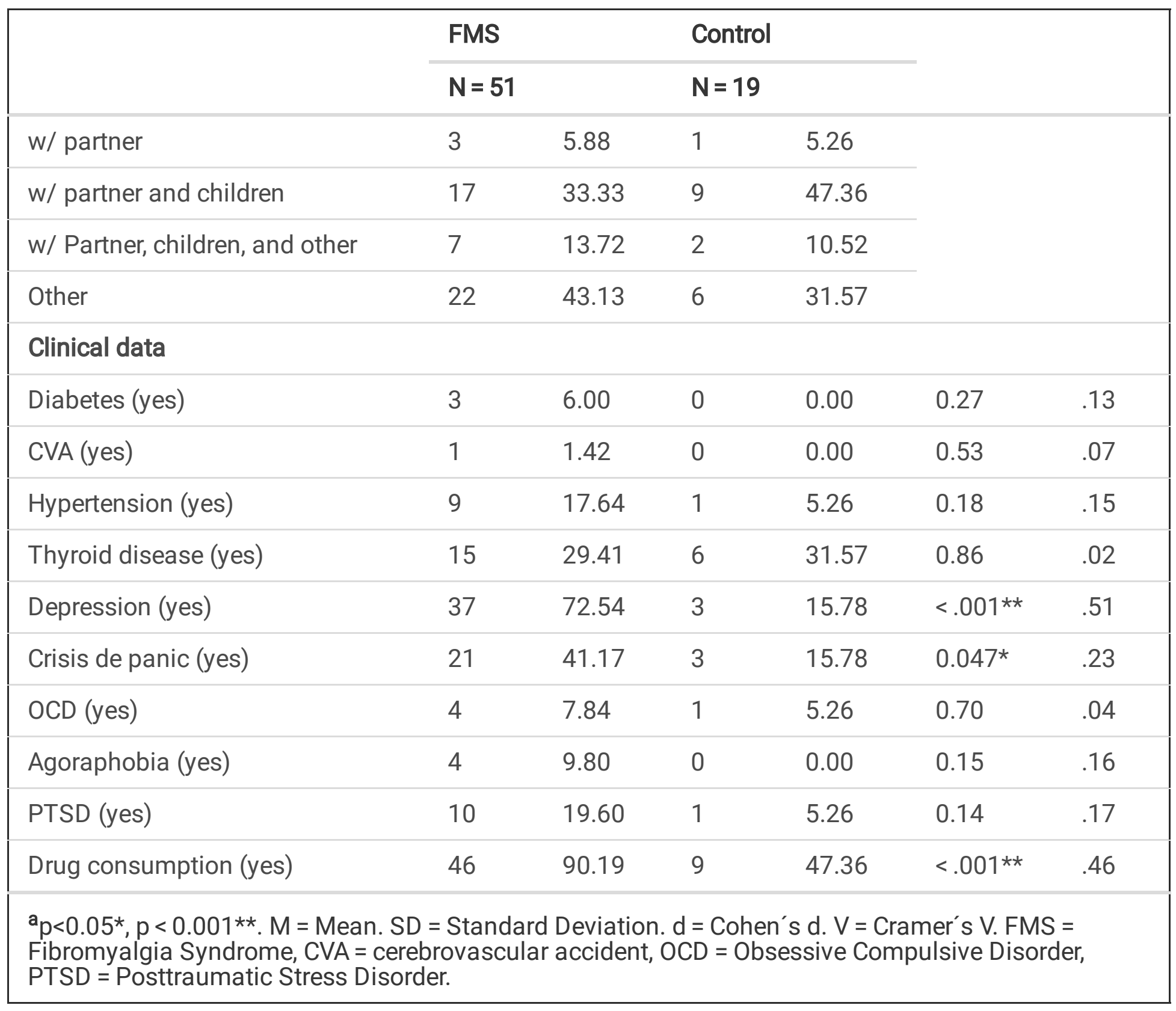

To analyze physical pain differences, difficulties due to the pandemic, mood, and their impact in FMS, we performed statistical comparisons showing that FMS patients exhibited higher levels of generalized physical pain, greater difficulties due to the pandemic in general and in several specific dimensions: mood, pain, financial, family, health, food supply. Table 2 summarizes the statistical comparisons in physical pain, difficulties due to the pandemic, mood, and their impact on FMS. 
Table 2

Physical pain, Difficulties due to the Pandemic, Mood, and their impact on FMS a

\begin{tabular}{|c|c|c|c|c|c|c|}
\hline \multirow{3}{*}{ Physical pain } & \multirow{2}{*}{$\begin{array}{l}\text { SFM } \\
N=51\end{array}$} & & \multicolumn{2}{|l|}{ Control } & \multirow[b]{3}{*}{$p$} & \multirow[b]{3}{*}{ d } \\
\hline & & & $N=19$ & & & \\
\hline & M & $\mathrm{DE}$ & $M$ & DE & & \\
\hline Head & 6.02 & 3.21 & 2.94 & 3.00 & $<.001^{\star *}$ & -.97 \\
\hline Nape & 6.37 & 3.58 & 1.15 & 2.34 & $<.001^{\star \star}$ & -1.58 \\
\hline Behind the neck & 7.31 & 2.90 & 1.78 & 2.34 & $<.001^{\star \star}$ & -1.94 \\
\hline Face & 3.45 & 3.34 & 0.36 & 0.95 & $<.001^{\star \star}$ & -1.05 \\
\hline Neck & 6.68 & 2.89 & 2.42 & 2.43 & $<.001 * \star$ & -1.59 \\
\hline Shoulders & 7.49 & 2.83 & 2.26 & 3.01 & $<.001^{\star \star}$ & -1.81 \\
\hline High back & 7.49 & 2.88 & 2.52 & 3.00 & $<.001^{\star \star}$ & -1.70 \\
\hline Middle back & 6.60 & 2.94 & 2.05 & 2.91 & $<.001 * \star$ & -1.55 \\
\hline Lower back & 7.39 & 2.96 & 2.78 & 2.99 & $<.001^{\star \star}$ & -1.54 \\
\hline Shoulders & 7.49 & 2.83 & 2.26 & 3.01 & $<.001 * \star$ & -1.81 \\
\hline Hands & 6.90 & 3.30 & 1.05 & 2.24 & $<.001^{\star *}$ & -1.91 \\
\hline Wrist & 6.60 & 3.00 & 1.05 & 1.47 & $<.001^{\star *}$ & -2.06 \\
\hline Chest & 3.82 & 3.63 & 0.57 & 1.46 & $<.001^{\star *}$ & -1.01 \\
\hline Ribs & 3.76 & 3.45 & 0.52 & 1.61 & $<.001^{\star *}$ & -1.05 \\
\hline Hips & 6.54 & 3.44 & 1.57 & 2.52 & $<.001^{\star \star}$ & -1.54 \\
\hline Thighs & 6.17 & 3.50 & 0.78 & 2.14 & $<.001^{\star *}$ & -1.68 \\
\hline Buttocks & 4.94 & 3.33 & 1.26 & 2.62 & $<.001 \star \star$ & -1.16 \\
\hline Knees & 6.45 & 3.31 & 1.89 & 2.97 & $<.001^{\star *}$ & -1.41 \\
\hline Tibias & 5.17 & 3.76 & 0.26 & 0.80 & $<.001 * \star$ & -1.50 \\
\hline Shinbone & 5.35 & 3.83 & 0.26 & 0.80 & $<.001^{\star *}$ & -1.53 \\
\hline Feet & 5.76 & 3.49 & 0.73 & 1.24 & $<.001^{\star *}$ & -1.64 \\
\hline Total pain & 113.64 & 45.36 & 25.89 & 25.43 & $<.001 * \star$ & -2.13 \\
\hline
\end{tabular}

${ }^{a} p<0.05^{\star}, p<0.001^{\star \star} . M=$ Mean. SD = Standard Deviation. $d=$ Cohen's d. HAD = Hospital Anxiety and Depression Scale. 


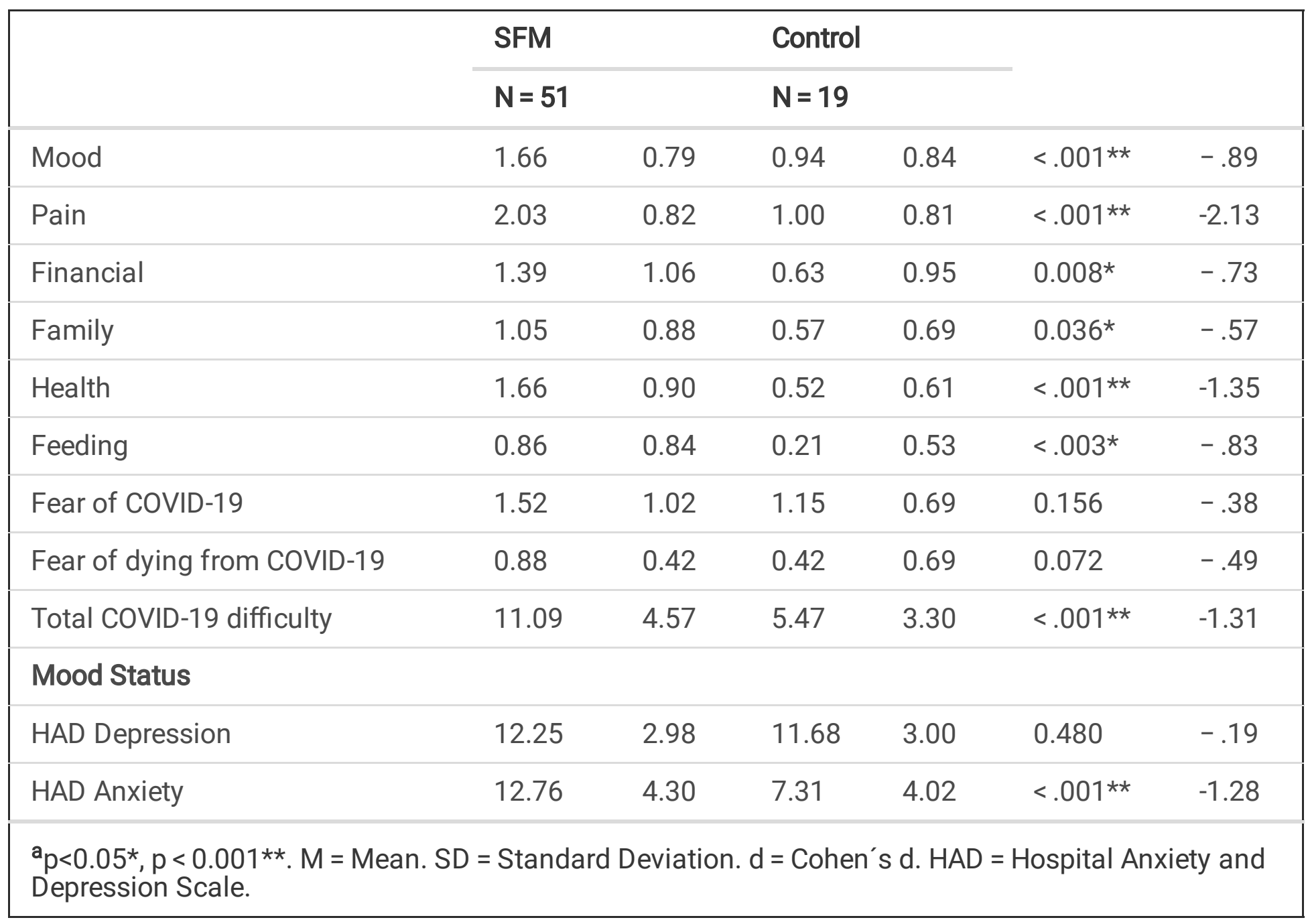

Cognitive performance was evaluated in different domains. Interestingly, we found no differences in memory performance and phonological fluency. However, the executive functions exhibited impairment in the FMS group. Specifically, the semantic fluency and the attention measured using the Symbol-Digit test. Additionally, the executive functions evaluated using the self-rating Dysexecutive Questionnaire (DEX-Sp) also showed lower performance in all dimensions: impulsivity and disorganization (Fig. 3). Table 3 shows the statistical comparisons between groups in cognitive performance. 
Table 3

Cognitive performance and dysexecutive symptoms standardized by age and education (z-score) a

\begin{tabular}{|c|c|c|c|c|c|c|}
\hline \multirow{3}{*}{ Memory } & \multirow{2}{*}{\multicolumn{2}{|c|}{$\begin{array}{l}\text { FMS } \\
\mathrm{N}=51\end{array}$}} & \multirow{2}{*}{\multicolumn{2}{|c|}{$\begin{array}{l}\text { Control } \\
\mathrm{N}=19\end{array}$}} & \multirow[b]{3}{*}{$\mathbf{p}$} & \multirow[b]{3}{*}{ d } \\
\hline & & & & & & \\
\hline & M & DE & M & DE & & \\
\hline HVLT Learning & -0.51 & 0.93 & -0.28 & 0.64 & 0.330 & .42 \\
\hline HVLT Recall & -0.38 & 0.94 & -0.02 & 1.02 & 0.171 & .37 \\
\hline \multicolumn{7}{|l|}{ Language } \\
\hline Phonological Fluency & 0.86 & 1.20 & 1.11 & 1.17 & 0.442 & .20 \\
\hline Semantic Fluency & -0.00 & 1.14 & 1.19 & 0.65 & $<.001^{\star *}$ & 1.15 \\
\hline \multicolumn{7}{|l|}{ Executive Functions } \\
\hline SDMT & -1.45 & 0.70 & -0.67 & 1.16 & $<.001^{\star \star}$ & .91 \\
\hline Digit Span Test Forward & -1.28 & 0.82 & -0.92 & 0.63 & 0.084 & .47 \\
\hline Digit Span Test Inverse & -0.57 & 0.89 & -0.32 & 0.83 & 0.289 & .28 \\
\hline DEX Disorganization & 2.34 & 1.53 & -0.01 & 0.87 & $<.001^{\star \star}$ & -1.70 \\
\hline DEX Impulsivity & 1.47 & 1.41 & -0.08 & 1.17 & $<.001^{\star \star}$ & -1.14 \\
\hline DEX Dysexecution total & 2.14 & 1.55 & -0.05 & 1.06 & $<.001^{\star \star}$ & -1.52 \\
\hline
\end{tabular}

To determine whether there is a relationship between time since diagnosis, anxiety score, depression score, the impact of FMS, pain rating, difficulties due to COVID-19, DEX-sp, SDMT, and FVS, we performed Pearson's $r$ correlations between these parameters. We found various correlations (Table 4). 
Table 4

Correlations between Time Since FMS Diagnosis, Anxiety, Depression, Impacto n FMS, Pain, Difficulties due to the Pandemic, DEX, SDMT, and SVFT .

\begin{tabular}{|c|c|c|c|c|}
\hline & DEX total & SDMT Z score & FVS Z score & Tiempo Dg. FMS \\
\hline Time since FMS diagnosis & $.59 * \star$ & $-.34 * \star$ & $-.44^{\star \star}$ & - \\
\hline HAD Anxiety & $.63^{\star \star}$ & $-.28 *$ & $-.33^{*}$ & $.61 * \star$ \\
\hline HAD Depression & .05 & .15 & .06 & .05 \\
\hline Total Pain & $.67 * \star$ & $-.38 * \star$ & $-.43^{\star \star}$ & $.67 * \star$ \\
\hline Total COVID-19 difficulty & $.55^{\star \star}$ & $-.29 *$ & -.14 & $.55 \star \star$ \\
\hline
\end{tabular}

Afterward, in order to determine the effect of the difficulties due to the pandemic, physical pain, and psychological distress over cognitive performance, we performed a stepwise linear regression. Importantly, we found that the predictor variables have a significant effect on cognitive performance (Table 5). 
Table 5

Stepwise Linear Regression. Pain, Anxiety, and Fibromyalgia explaining cognitive performance ${ }^{a}$. Total sample $(\mathrm{N}=70)$

SDMT Z-score

$\begin{array}{lllll}\beta & t & p & R^{2} & \Delta R^{2}\end{array}$

Model 1

Total Pain

$-.38$

$-3.44$

$<.001^{\star \star}$

.13

DEX Total Z-score

$\begin{array}{lllll}\beta & t & p & R^{2} & \Delta R^{2}\end{array}$

Model 1

HAD Anxiety

.70

8.07

$<.001^{\star \star}$

.48

Model 2

$\begin{array}{llllll}\text { HAD Anxiety } & .55 & 5.86 & <.001^{\star \star} & .53 & .06 \\ \text { Fibromyalgia } & .28 & 3.04 & <.003^{\star} & & \end{array}$

SVFT Z-score

$\begin{array}{lllll}\beta & t & p & R^{2} & \Delta R^{2}\end{array}$

Model 1

Fibromyalgia

$-.46$

$-4.29$

$<.001^{\star \star}$

.20

${ }^{a} p<0.05^{\star}, p<0.001^{\star *}$. HAD $=$ Hospital Anxiety and Depression Scale. SDMT = Symbol Digits Modalities Test. DEX = Disexecutive Questionnaire. SVFT = Semantic Verbal Fluency Test.

\section{Discussion}

In this study, the cognitive performance of FMS patients was evaluated to determine whether cognitive functions were affected by physical pain and/or psychological distress due to the pandemic. To address this question, we designed a novel online protocol that can be reproduced for clinical purposes. We found impairment in executive functions or high-order cognitive functions for the FMS group, where the physical pain explained part of this significant result. This finding was in line with our predictions since it has previously been reported that physical pain reduces attention capacity, affecting overall cognitive performance ${ }^{20}$, which agrees with the Neurocognitive Model of Attention to Pain ${ }^{29}$.

Furthermore, FMS patients exhibited semantic fluency impairment suggesting semantic access difficulties, which involves executive action to retrieve information from long-term memory ${ }^{30}$. This result 
is in line with studies showing deficits in this area ${ }^{31}$. Additionally, we have found for the first time an association between dysexecutive symptoms, pain, time since diagnosis of FMS, difficulties due to the pandemic, and anxiety symptoms.

This study has some limitations. Firstly, we evaluated only women from an urban context; thus, these findings are not extrapolatable to other groups, such as men with FMS or people from rural zones. Secondly, Results involving the difficulties due to pandemic evaluation have to be taken with precautions since these were conducted without previous psychometric properties data of this instrument, which might affect the level of reliability of this particular finding. Finally, we strongly recommend for future research including other social groups (for instance, men and rural groups) to strengthen the results and subsequent interpretations.

\section{Conclusions}

Our results show that Chilean women with Fibromyalgia exhibit subjective and objective cognitive impairments, predominantly in executive functions or high cognitive demanding tasks. These impairments are mainly explained by pain and anxiety symptoms that they suffer, which are positively associated with the difficulties experienced from the COVID-19 pandemic. Additionally, these results express the relevance of considering the treatment of pain and anxiety symptoms as a therapeutic target for cognitive impairments and the effect of the pandemic on their daily functioning.

\section{Methods}

This study is case-control, cross-sectional, descriptive, and non-experimental. The sampling technique was non-probabilistic. All the procedures were carried out remotely in tele-neuropsychology format ${ }^{32}$ due to the health emergency caused by COVID-19 pandemic. The ethics committee of the Universidad Santo Tomás approved procedures, and all methods were carried out in accordance with the relevant guidelines and regulations.

\section{Subjects}

Data was collected from seventy right-handed and Chilean adult women. From the total sample, there was a group of fifty women diagnosed with FMS (mean age $=44.05, \mathrm{SD}=8.71$ ) and nineteen healthy women as a paired-match (sex, age, nationality, demographic similarities) control group (mean age = $40.63, \mathrm{SD}=9.79$ ). The sample size followed the gold standard reported in the meta-analysis by Bell et al.

19 . Patients with FMS were diagnosed by a specialist following the 1990 American College of Rheumatology criteria for FMS ${ }^{28}$ and were recruited from Fibromyalgia Associations located in distributed regions of Chile, while participants belonging to the control group were recruited based on demographic similarities with patients. Exclusion criteria for the control group were neurological or psychiatric disorders and medical background of severe somatic diseases. All participants signed an informed consent form before the beginning of the study. 


\section{Instruments}

- Sociodemographic Questionnaire: We designed an open-ended and multiple options questionnaire instrument that measures general demographic characteristics (age, medications, illnesses, schooling, history of mental disorders, medical background, among other aspects). Additionally, in this questionnaire a likert scale was included to evaluate difficulties due to the pandemic (mood, physical pain, financial problems, family, health, fear of being infected, food, and fear of dying).

- Hospital Anxiety and Depression Scale (HAD) ${ }^{33}$ : The HAD is a self-assessment scale that detects states of depression and anxiety in patients with different chronic conditions, assessing the cognitive and behavioral symptoms of anxiety and depression. It is composed of two subscales (Depression and Anxiety), each of them has 7 items. Each score varies between 0 to 21. The response options range from: absence $/$ minimum presence $=0$ to maximum presence $=3$. The higher the score obtained, the greater the intensity or severity of the symptoms. This scale has been validated in patients with FMS from a Spanish population and has good psychometric properties ${ }^{33}$.

- Disexecutive Questionnaire (DEX-Sp) ${ }^{34}$ : The DEX-sp is a brief and easy to complete Likert-type selfreport test with twenty items, and five response options between 'never' ( 0 points) and 'very often' (4 points) used to estimate executive dysfunction. This test assesses abstract thinking, planning, insight, temporal sequencing, impulse control, response inhibition, decision-making, as well as the presence of fables, impulsivity, euphoria, apathy, aggressiveness, restlessness motor, superficial affective responses, perseverance, distractibility, and disregard for social rules ${ }^{35}$. This scale presents good psychometric properties and has been validated in the Spanish-speaking population ${ }^{34}$.

Visual Analog Scale of Pain (VAS): The VAS is a subjective measure of acute and chronic pain intensity. Scores are recorded by making a handwritten mark on a 10-cm line representing a continuum between "no pain" and " the worst pain imaginable." The VAS is a reliable and valid scale for many patient populations ${ }^{36}$. A value lower than 4 on the VAS means mild or mild-moderate pain, while a value between 4 and 6 implies moderate-severe pain. A value higher than 6 implies the presence of very intense pain.

\section{Cognitive variables}

- Hopkins Verbal Learning Test (HVLT) ${ }^{37}$ : The HVLT is a verbal memory test consisting of a 12-item word list composed of 3 semantic categories, which subjects should recall after the list has been read to them. This procedure is repeated three times. 25 minutes later, subjects are asked to recall the list of words again. Then, for a yes/no recognition, a list of 24 words is read, which consists of the 12 original/target words, 6 distractors from the same semantic categories and 6 unrelated distractors. This test provides scores for a learning curve, long-term memory, and recognition/discrimination. The HVLT is a valid instrument in the Latin American Spanish-speaking adult population with good psychometric properties 37. 
- Symbol Digit Modalities Test (SDMT): The SDMT measures sustained attention and speed of information processing ${ }^{38}$ consisting of nine digits paired with symbols underneath. Participants are asked to provide the corresponding number for each symbol for $90 \mathrm{~s}$. The SDMT is a valid instrument in the Latin American Spanish-speaking adult population with good psychometric properties ${ }^{39}$.

F-A-S Test: These tests evaluate executive functions since they demand participants to be flexible, organize information, provide effort, and control inhibition when it is needed ${ }^{40}$. In order to evaluate these complex cognitive functions, we used the phonological and semantic fluency tasks, which both have been validated and normalized in the Latin American Spanish-speaking adult population with good psychometric properties ${ }^{40}$.

- Phonological Verbal Fluency: This test aims to ask participants to create as many words as possible, beginning with a specific letter $(F, A, S)$ for 60 seconds. If a participant does not respond within 10 seconds, the examiner gives prompts ${ }^{40}$.

- Semantic Verbal Fluency: This test demands participants to produce items that belong to a given category (e.g., animals) within 60 seconds. The participants are also provided with prompts for this test when they do not respond within 10 seconds ${ }^{40}$.

Digit Span tests: These are verbal tasks, with stimuli presented auditorily. These tests aim to assess attention (Digit Span Forward) and working memory capacity (Digit Span Reverse) ${ }^{41}$.

- Digit Span Forward: In this test, the experimenter says a sequence of digits (one digit per second); at the end, the participant is asked to repeat the digits back to the experimenter in the same order that was given. The task begins with a few digit sequences, and then the number of digits increases ${ }^{41}$.

- Digit Span Reverse: This version of the test has the same structure as the Digit Span Forward. The difference is that the participant is asked to repeat the digits in reverse order which requires manipulation of information, thus working memory function ${ }^{41}$.

\section{Procedure}

After the ethics committee of the Universidad Santo Tomás approved the research project, this was formally presented at the Fibromyalgia Association of Chile. Women diagnosed with FMS members of the association along the country were voluntarily recruited. Afterward, patients were contacted to confirm computer/tablet or smartphone availability with an internet connection. Subsequently, they received an online form that included a brief description of the research, informed consent, sociodemographic questionnaire, medical history, and the instruments: difficulties due to the pandemic, the DEX-Sp, VAS, and HAD scales.

After the first data collection, the second part of the process was to conduct the online neurocognitive protocol consisting of the HVLT, SDMT, F-A-S, and Digit Span tests. The evaluation protocol lasted 
approximately 30 minutes and was applied through the Whereby platform from March to May 2021.

Later, the control group with similar characteristics as the FMS patient group was recruited. The control group was evaluated following the same procedure aforementioned. Finally, once all data were collected, statistical analyses were performed (Figure 2).

\section{Data Analysis}

The student's t-test was used to compare variables between groups. Additionally, Cohen's $d$ test was performed to estimate the size effect. Moreover, we conducted the Chi square test and the Cramer's V test for nominal variables, respectively. To determine the association level between variables, we used the Pearson $r$ correlation test. Furthermore, in order to determine the effect of pandemic difficulties, physical pain, and psychological distress on cognitive performance, a stepwise linear regression was used, including 5000 samples bootstrap. All statistical analyzes were performed using the SPSS 25 statistical software synchronized with JASP, considering a significance threshold of $p<0.05$.

\section{Declarations}

\section{Data availability}

The data of the current study are available from the authors upon reasonable request.

\section{Acknowledgments}

We want to thank the Fibromyalgia Association Chile (Asociación Fibromialgia Chile) for kindly helping us in contacting their members.

\section{Author Contributions}

M.R.-H.: Conceived the project, evaluation of subjects, analyzed data, prepared figures, wrote manuscript, revised and commented the manuscript. I.T.-R.: Analyzed data, prepared figures, discussed data. R.A.-C.: Evaluation of subjects, analyzed data, wrote manuscript, revised and commented the manuscript. D.P.: Evaluation of subjects, wrote manuscript, discussed data, prepared figures, revised and commented manuscript. M.S.-A.: Analyzed data, prepared figures, discussed data. N.L.: Analyzed data, prepared figures, discussed data. M.D.-S.: Analyzed data, prepared figures, wrote manuscript, discussed data, revised and commented the manuscript.

\section{Competing interests}

The authors declare that they have no competing interests.

\section{References}


1. Bennett, R. M. Clinical manifestations and diagnosis of fibromyalgia. Rheumatic Disease Clinics, 35 (2), 215-232 https://doi.org/10.1016/j.rdc.2009.05.009 (2009).

2. Schmidt-Wilcke, T. \& Diers, M. New insights into the pathophysiology and treatment of fibromyalgia. Biomedicines, 5 (2), 22 https://doi.org/10.3390/biomedicines5020022 (2017).

3. Queiroz, L. P. Worldwide epidemiology of fibromyalgia. Current pain and headache reports, 17 (8), 356 https://doi.org/10.1007/s11916-013-0356-5 (2013).

4. Jones, G. T. et al. The prevalence of fibromyalgia in the general population: a comparison of the American College of Rheumatology 1990, 2010, and modified 2010 classification criteria. Arthritis \& rheumatology, 67 (2), 568-575 https://doi.org/10.1002/art.38905 (2015).

5. Juuso, P., Skär, L., Olsson, M. \& Söderberg, S. Meanings of feeling well for women with fibromyalgia. Health care for women international, 34 (8), 694-706 https://doi.org/10.1080/07399332.2012.736573 (2013).

6. MINSAL. Orientación Técnica Abordaje de la Fibromialgia 1-05-2016 https://rehabilitacion.minsal.cl/wp-content/uploads/2016/06/OT-Fibromialgia-2016.pdf

7. Bradley, L. A. Pathophysiology of fibromyalgia. The American journal of medicine, 122 (12), S22S30 https://doi.org/10.1016/j.amjmed.2009.09.008 (2009).

8. Semiz, M. et al. Post-Traumatic Stress Disorder, Alexithymia and Somatoform Dissociation in Patients with Fibromyalgia. Turkish Journal of Physical Medicine \& Rehabilitation/Turkiye Fiziksel Tip ve Rehabilitasyon Dergisi, 60 (3), https://doi.org/10.5152/tftrd.2014.48254 (2014).

9. Campos, R. P. \& Vázquez, M. I. R. Health-related quality of life in women with fibromyalgia: clinical and psychological factors associated. Clinical rheumatology, 31 (2), 347-355 (2012).

10. Lee, J. W. et al. Determinants of quality of life in patients with fibromyalgia: A structural equation modeling approach. Plos one, 12 (2), e0171186 https://doi.org/10.1371/journal.pone.0171186 (2017).

11. Palstam, A. \& Mannerkorpi, K. Work ability in fibromyalgia: an update in the 21 st century. Current rheumatology reviews, 13 (3), 180-187 https://doi.org/10.2174/1573397113666170502152955 (2017).

12. Kravitz, H. M. \& Katz, R. S. Fibrofog and fibromyalgia: a narrative review and implications for clinical practice. Rheumatology international, 35 (7), 1115-1125 https://doi.org/10.1007/s00296-014-32087 (2015).

13. Katz, R. S., Heard, A. R., Mills, M. \& Leavitt, F. The prevalence and clinical impact of reported cognitive difficulties (fibrofog) in patients with rheumatic disease with and without fibromyalgia. J. Clin. Rheumatol, 10, 53-58 https://doi.org/10.1097/01.rhu.0000120895.20623.9f (2004).

14. Montoro, C. I., Duschek, S., Muñoz Ladrón de Guevara, C. \& Fernández-Serrano, M. J. \& Reyes del Paso, G. A. Aberrant cerebral blood flow responses during cognition: Implications for the understanding of cognitive deficits in fibromyalgia., 29 (2), 173 https://doi.org/10.1037/neu0000138 (2015). 
15. Kalfon, T. B. O., Gal, G., Shorer, R. \& Ablin, J. N. Cognitive functioning in fibromyalgia: the central role of effort. Journal of Psychosomatic Research, 87, 30-36 https://doi.org/10.1016/j.jpsychores.2016.06.004 (2016).

16. Cherry, B. J. et al. Cognitive performance in women aged 50 years and older with and without fibromyalgia. Journals of Gerontology Series B: Psychological Sciences and Social Sciences, 69 (2), 199-208 https://doi.org/10.1093/geronb/gbs122 (2014).

17. Verdejo-García, A., López-Torrecillas, F., Calandre, E. P., Delgado-Rodríguez, A. \& Bechara, A. Executive function and decision-making in women with fibromyalgia. Archives of Clinical Neuropsychology, 24 (1), 113-122 https://doi.org/10.1093/arclin/acp014 (2009).

18. Gelonch, O., Garolera, M., Valls, J., Rosselló, L. \& Pifarré, J. Cognitive complaints in women with fibromyalgia: are they due to depression or to objective cognitive dysfunction? Journal of clinical and experimental neuropsychology, 39 (10), 1013-1025 https://doi.org/10.1080/13803395.2017.1301391 (2017).

19. Bell, T. et al. Meta-analysis of cognitive performance in fibromyalgia. Journal of clinical and experimental neuropsychology, 40 (7), 698-714 https://doi.org/10.1080/13803395.2017.1422699 (2018).

20. Teodoro, T., Edwards, M. J. \& Isaacs, J. D. A unifying theory for cognitive abnormalities in functional neurological disorders, fibromyalgia and chronic fatigue syndrome: systematic review. Journal of Neurology, Neurosurgery \& Psychiatry, 89 (12), 1308-1319 https://doi.org/10.1136/jnnp-2017317823 (2018).

21. Wu, Y. L., Huang, C. J., Fang, S. C., Ko, L. H. \& Tsai, P. S. Cognitive impairment in fibromyalgia: A metaanalysis of case-control studies. Psychosomatic medicine, 80 (5), 432-438 https://doi.org/10.1097/PSY.0000000000000575 (2018).

22. Schmidt-Wilcke, T. \& Diers, M. New insights into the pathophysiology and treatment of fibromyalgia. Biomedicines, 5 (2), 22 https://doi.org/10.3390/biomedicines5020022 (2017).

23. Semiz, M. et al. Post-Traumatic Stress Disorder, Alexithymia and Somatoform Dissociation in Patients with Fibromyalgia. Turkish Journal of Physical Medicine \& Rehabilitation/Turkiye Fiziksel Tip ve Rehabilitasyon Dergisi, 60 (3), https://doi.org/10.5152/tftrd.2014.48254 (2014).

24. Galvez-Sánchez, C. M., Duschek, S. \& Del Paso, G. A. R. Psychological impact of fibromyalgia: current perspectives. Psychology research and behavior management, 12, 117 https://doi.org/10.2147/prbm.s178240 (2019).

25. Dubey, M. J. et al. COVID-19 and addiction. Diabetes \& Metabolic Syndrome: Clinical Research \& Reviews, 14 (5), 817-823 https://doi.org/10.1016/j.dsx.2020.05.035 (2020).

26. Serrano-lbáñez, E. R., Esteve, R., Ramírez-Maestre, C., Ruiz-Párraga, G. T. \& López-Martínez, A. E. Chronic pain in the time of COVID-19: Stress aftermath and central sensitization. British Journal of Health Psychology, 26 (2), 544-552 https://doi.org/10.1111/bjhp.12483 (2020).

27. Rubin, R., Abbasi, J. \& Voelker, R. Latin America and Its Global Partners Toil to Procure Medical Supplies as COVID-19 Pushes the Region to Its Limit. JAMA, 324 (3), 217-219 
https://doi.org/10.1001/jama.2020.11182 (2020).

28. Wolfe, F. et al. Diagnosis of Fibromyalgia: Disagreement Between Fibromyalgia Criteria and ClinicianBased Fibromyalgia Diagnosis in a University Clinic. Arthritis Care Res, 71 (3), 343-351 https://doi.org/10.1002/acr.23731 (2019).

29. Legrain, V. et al. A neurocognitive model of attention to pain: behavioral and neuroimaging evidence., 144, 230-232 https://doi.org/10.1016/j.pain.2009.03.020 (2009).

30. Fisk, J. E. \& Sharp, C. A. Age-related impairment in executive functioning: Updating, inhibition, shifting, and access. Journal of Clinical and Experimental Neuropsychology, 26 (7), 874-890 (2004).

31. Di Tella, M. et al. Theory of Mind and Emotional Functioning in Fibromyalgia Syndrome: An Investigation of the Relationship between Social Cognition and Executive Function. PloS One, 10 (1), 1-16 (2015).

32. Bilder, R. M. et al. InterOrganizational practice committee recommendations/guidance for teleneuropsychology (TeleNP) in response to the COVID-19 pandemic. Clin. Neuropsychol, 34 (7-8), 1314-1334 https://doi.org/10.1080/13854046.2020.1767214 (2020).

33. Cabrera, V., Martín-Aragón, M., Terol, M. C., Núñez, R. \& de Pastor, M. los Á. La Escala de Ansiedad y Depresión Hospitalaria (HAD) en fibromialgia: Análisis de sensibilidad y especificidad. Terapia Psicológica, 33(3), 181-193, doi:10.4067/s0718-48082015000300003 (2015)

34. Pedrero Pérez, E. J. et al. J. C. B., et al. Versión española del Cuestionario Disejecutivo (DEX-Sp): Propiedades psicométricas en adictos y población no clínica. Adicciones, 21 (2), 155 https://doi.org/10.20882/adicciones.243 (2009).

35. Norris, G. \& Tate, R. L. The Behavioural Assessment of the Dysexecutive Syndrome (BADS): Ecological, Concurrent and Construct Validity. Neuropsychological Rehabilitation, 10 (1), 33-45 (2000).

36. Karcioglu, O., Topacoglu, H., Dikme, O. \& Dikme, O. A systematic review of the pain scales in adults: which to use? The American journal of emergency medicine, 36 (4), 707-714 (2018).

37. Arango-Lasprilla, J. C. et al. Hopkins Verbal Learning Test- Revised: Normative data for the Latin American Spanish speaking adult population. NeuroRehabilitation, 37 (4), 699-718 https://doi.org/10.3233/NRE-151286 (2015a).

38. Spreen, O. \& Strauss, E. A compendium of neuropsychological tests: administration, norms, and commentary (Oxford University Press, 1998).

39. Arango-Lasprilla, J. C. et al. Symbol Digit Modalities Test: Normative data for the Latin American Spanish speaking adult population. NeuroRehabilitation, 37 (4), 625-638 https://doi.org/10.3233/NRE-151282 (2015b).

40. Olabarrieta-Landa, L. et al. Verbal fluency tests: Normative data for the Latin American Spanish speaking adult population. NeuroRehabilitation, 37 (4), 515-561 https://doi.org/10.3233/NRE151279 (2015).

41. García-Morales, P., Gich-Fullà, J., Guardia-Olmos, J. \& Peña-Casanova, J. Digit span, automatic speech and orientation: amplified norms of the Barcelona Test., 13 (6), 271-276 (1998). 


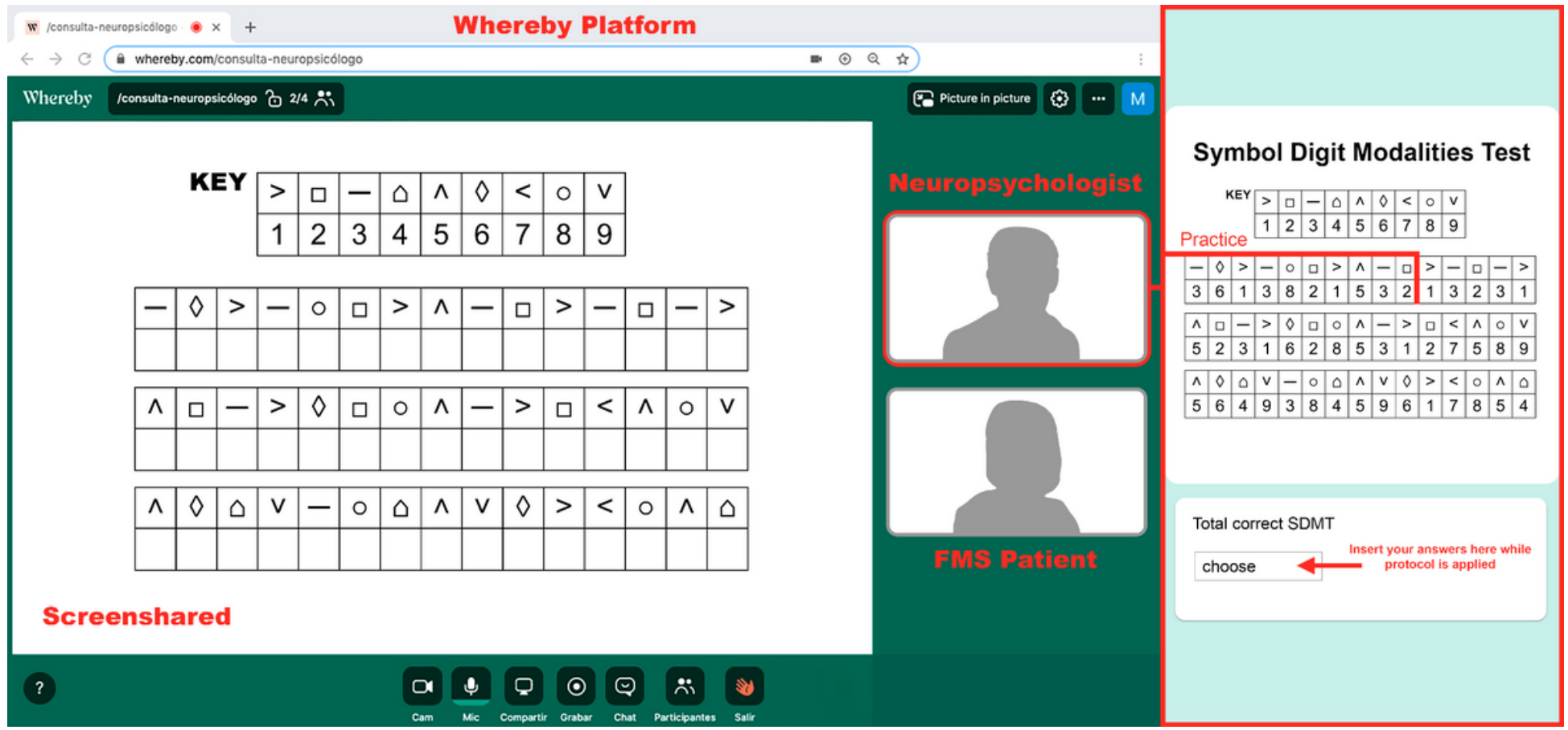

\section{Figure 1}

Representative neuropsychological online protocol. A trained neuropsychologist explained and guided the cognitive evaluation to FMS and control groups through the Whereby platform.

1)

Contact with the
Fibromyalgia
Association Chile.
Recruitment of FMS
patients

6)

Control group
recruitment and
reproduction of the
same evaluation
procedure

3)

online form and scale administration

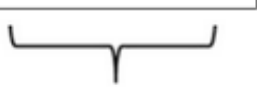

- Informed consent

- Sociodemographic Questionnaire

- DEX-Sp

- VAS

4)

Scheduling the online neurocognitive evaluation in teleneuropsychology format

Figure 2 
SDMT

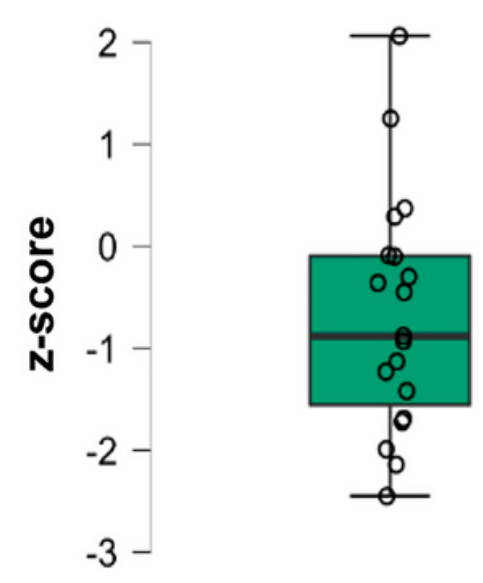

Control

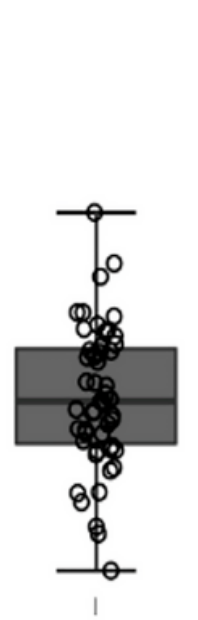

Fibromyalgia

\section{Semantic Fluency}

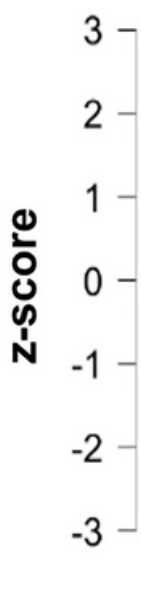

$-1-$
-2
$-3-$

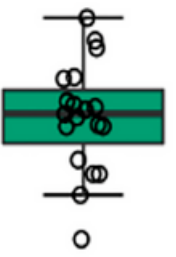

Control

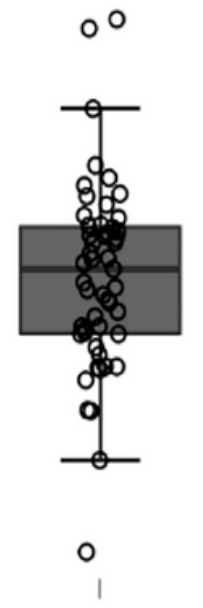

Fibromyalgia

\section{Figure 3}

Whisker and boxplots. Cognitive performance in Symbol Digit Modalities Test (SDMT) and Semantic Fluency test. The FMS group exhibited a significant decrease in their executive functioning performance in both measurements: SDMT (<.001**) and Semantic Fluency Test $\left(<.001^{\star \star}\right)$. 